\title{
Three-Dimensional Transmission Electron Microscopy: A Novel Imaging and Characterization Technique with Nanometer Scale Resolution for Materials Science
}

\author{
A. J. Koster, ${ }^{\dagger}$ U. Ziese, ${ }^{\dagger}$ A. J. Verkleij, ${ }^{\dagger}$ A. H. Janssen, ${ }^{\dagger}$ and K. P. de Jong* ${ }^{*}$ \\ Department of Molecular Cell Biology, Utrecht University, Padualaan 8, 3584 CH Utrecht, The Netherlands, \\ and Department of Inorganic Chemistry and Catalysis, Debye Institute, Utrecht University, Sorbonnelaan 16, \\ 3584 CA Utrecht, The Netherlands
}

Received: April 24, 2000; In Final Form: July 20, 2000

\begin{abstract}
Three-dimensional transmission electron microscopy (3D-TEM), effectuated by multiple imaging of a sample combined with image analysis, offers a new approach in materials science to obtain 3D information of complex solid materials. Here we report first-of-its-kind results that have been obtained with zeolite materials. Virtual cross-sections and volume rendering of the 3D reconstruction of a metal/zeolite crystal $(\mathrm{Ag} / \mathrm{NaY})$ give unequivocal information on the location of the silver particles (10-40 nm in diameter). Virtual cross-sections of the 3D reconstruction of an acid-leached mordenite show the three-dimensional mesoporous channel system $(3-20 \mathrm{~nm}$ in diameter) with a clarity and definition not seen before.
\end{abstract}

Nano-structured inorganic materials, such as solid catalysts, ${ }^{1}$ photonic crystals, ${ }^{2}$ and electronic devices, ${ }^{3}$ are of growing importance in various fields. To gain precision in synthesis and assembly of solids, knowledge of their three-dimensional (3D) structure is required. Electron microscopy is the only technique available to obtain structural information on these types of materials at nanometer scale resolution. However, scanning electron microscopy (SEM) provides surface information with limited information about the internal structure, whereas transmission electron microscopy (TEM) provides only 2D projections of the solid structure. Here we show that 3D-TEM offers a new approach in materials science to obtain 3D information, at nanometer scale resolution, of complex solid materials. 3DTEM, with its high potential as a unique tool to study biological structures, has rapidly developed during the past few years, which resulted in a first international workshop dedicated to the technique in $1997 .{ }^{4}$ Since then, a first generation of automated 3D-TEM data collection systems has become available to the scientific community to study macromolecular structures $^{5-7}$ as well as large cellular structures and organelles. ${ }^{8}$ So far, it was not recognized that 3D-TEM is also for materials science a unique and powerful tool in the investigation of individual samples of inorganic solids several hundred nanometers in size. With 3D-TEM one can study architecture and composition of individual samples with nanometer scale resolution. 3D-TEM complements other techniques generally used in the characterization of materials such as XRD, XPS, and highresolution TEM. In this paper we describe the principles of 3DTEM followed by two examples using zeolite materials. The first example involves establishing the location of metal particles in a metal-zeolite crystal $(\mathrm{Ag} / \mathrm{NaY})$; the second one involves imaging of mesopores in an acid-leached mordenite crystal.

Principles of 3D-TEM. With conventional TEM, images are acquired that are, to a first approximation, 2D projections of a

* Corresponding author. Fax: +31 30251 1027. E-mail: k.p.dejong@ chem.uu.nl.

$\dagger$ Department of Molecular Cell Biology, Utrecht University.

$\doteqdot$ Department of Inorganic Chemistry and Catalysis, Debye Institute, Utrecht University.
3D object. Electron tomography, ${ }^{9}$ here referred to as 3D-TEM, is a technique where a series of $2 \mathrm{D}$ projections (scattering contrast) is recorded that is subsequently used to compute a $3 \mathrm{D}$ image (3D reconstruction) of the object under investigation. These projections are obtained by recording $2 \mathrm{D}$ images at small angular increments while tilting the specimen over a wide angular range $\left( \pm 70^{\circ}\right)$. During the past few years, automated data collection systems were developed that enable direct recording of digital images and automatic compensation of image shifts and focus variations that occur because of the (mechanical) tilting of the specimen. ${ }^{10}$ With state-of-the-art equipment a series of 141 projections of a specimen-referred to as a data stack-is collected over an angular range of $140^{\circ}$. The subsequent 3D reconstruction is comprised of two steps: alignment of the data stack and reconstruction by backprojection. To achieve proper alignment, markers with sufficient contrast such as metal particles (e.g., $5 \mathrm{~nm}$ gold beads) are used. By least-squares fitting of the positions of these fiducial markers, the data series is aligned. After the alignment of the data series the $3 \mathrm{D}$ reconstruction has to be computed. The projection of a $3 \mathrm{D}$ object is equal to a central section of the Fourier transform of that object. A data series thus provides many different central sections of the Fourier transform of the sample, thus filling the 3D Fourier space. By inverse Fourier transform of the obtained 3D Fourier space a 3D image of the original object is obtained. An algorithm that is often used for the computation of the 3D reconstruction is resolution-weighted back-projection. ${ }^{9,10}$ The resolution $d$ of the 3D image can be approximated by $d=$ $\pi(T / N)$, where $N$ is the number of projections and $T$ the thickness of the sample. ${ }^{9,10}$

\section{Results and Discussion}

Metal/zeolite systems are key catalytic materials in applications such as hydro-isomerization of alkanes and hydrocracking of heavy petroleum fractions. One of the features of zeolites is the molecular dimension of the micropores that induces shapeselectivity effects in catalytic conversion reactions. The location of the metal function inside or outside the micropores can strongly affect these selectivity effects. To steer catalyst 


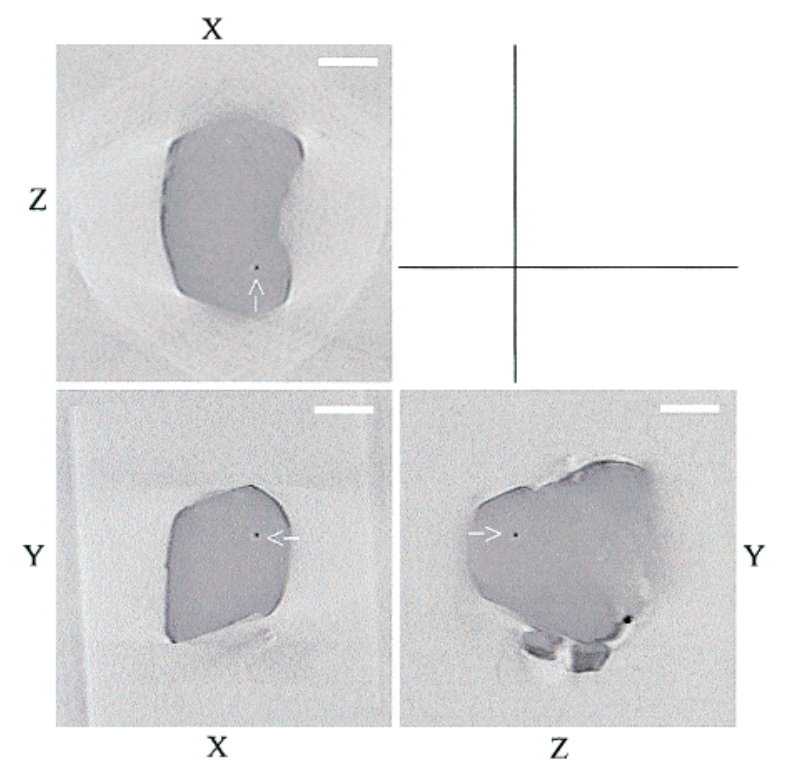

Figure 1. Three orthogonal crosscuts, with a thickness of $1.5 \mathrm{~nm}$, through the 3D-reconstruction of an $\mathrm{Ag} / \mathrm{NaY}$ crystal (500 nm thickness). The white arrows point to the same silver particle (18 nm diameter) that is located inside the zeolite. Scale bar is $200 \mathrm{~nm}$.

synthesis, knowledge on the location of the metal function is crucial. ${ }^{11,12}$ Bovin and co-workers ${ }^{13}$ have carried out TEM work on physical tomography of $\mathrm{Pt} /$ zeolite by ultramicrotomy of resinembedded crystals that enabled them to deduce the internal structure of the sample by imaging consecutive ultrathin $(20$ $\mathrm{nm}$ ) sections. Instead of physically cutting a crystal into slices we applied 3D-TEM and obtained truly 3D information on intact $\mathrm{Ag} /$ zeolite crystals by digital cross-sectioning.

$\mathrm{Ag} / \mathrm{NaY}$ was prepared by ion exchange of zeolite $\mathrm{NaY}$ (LZY54, UOP, Si/Al ratio 2.5) with $\mathrm{AgNO}_{3}$, after which the sample was dried at $423 \mathrm{~K}$ in argon and subsequently reduced at 423 $\mathrm{K}$ in $\mathrm{H}_{2}$. For electron microscopy, samples were mounted on a "perforated carbon"-coated copper grid by drying a droplet of a suspension of ground sample in ethanol on the grid. From the lattice diffraction images before and after $30 \mathrm{~min}$ of electron beam irradiation it was clear that no electron beam damage had occurred. From a representative $\mathrm{Ag} / \mathrm{NaY}$ crystal a tilt series of 143 images was taken from $+70^{\circ}$ to $-72^{\circ}$ with $1^{\circ}$ intervals at a magnification of $11.5 \mathrm{k}$ on a Philips CM 200 FEG microscope operated at $200 \mathrm{kV}$ with a $1024 \times 1024 \mathrm{CCD}$ camera (pixel size $1.12 \mathrm{~nm}$ ). For alignment purposes 7 silver particles on the zeolite crystallite were chosen as markers. From the data stack a reconstruction of $1150 \times 1150 \times 1150 \mathrm{~nm}$ with a resolution of $11 \mathrm{~nm}$ was calculated. This reconstruction can be visualized with slices through the reconstruction. Figure 1 shows three orthogonal slices through the reconstruction, all three showing the same silver particle (18 $\mathrm{nm}$ in diameter) that is located inside the zeolite crystallite. In Figure 2a,b the same zeolite crystallite is visualized with volume rendering, showing $10-40 \mathrm{~nm}$ silver particles on the external surface as well as a silver particle inside the zeolite. The colors were obtained by selecting two bands of gray-values (corresponding to the silver particles and the zeolite, respectively) and assigning different colors to the different bands. Figures 1 and 2 demonstrate that 3D-TEM enables an unequivocal determination of the location of metal particles in the interior or on the surface of 3D solid objects. Moving images of the aligned data stack and reconstruction are available as Supporting Information. Because 3D-TEM uses intact crystals, no deformation of the crystal took place as was the case with ultramicrotomy. ${ }^{13}$ The size of the silver particles in the $3 \mathrm{D}$ -
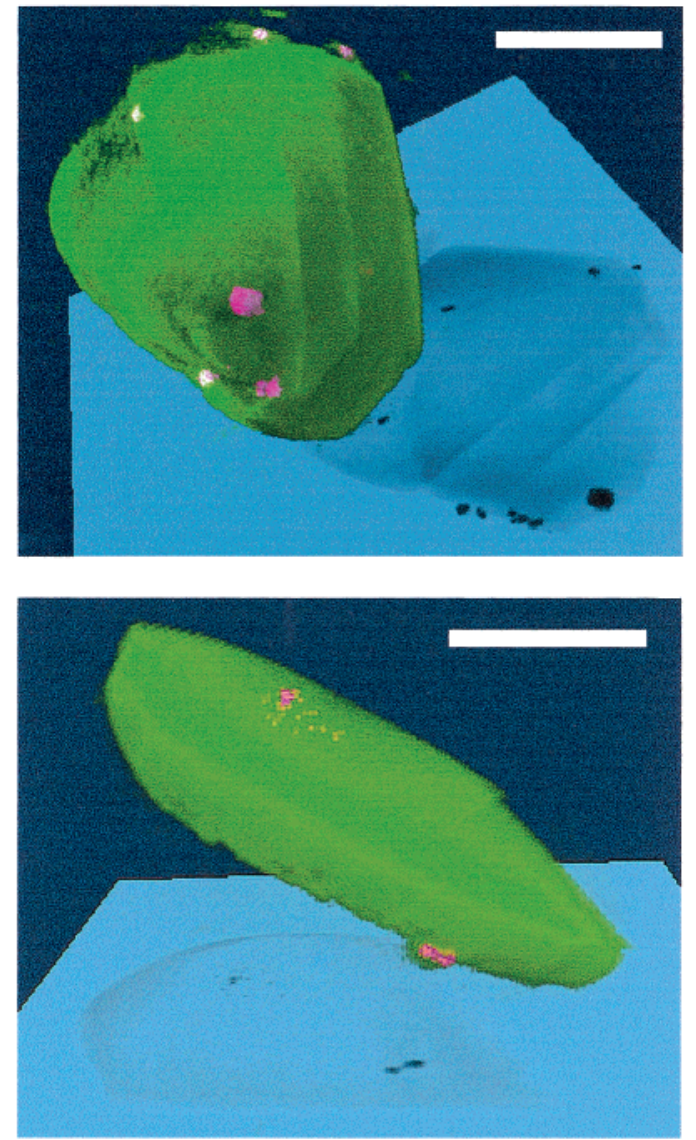

Figure 2. Volume rendering of the $\mathrm{Ag} / \mathrm{NaY}$ crystallite also shown in Figure 1. Silver particles are colored pink; the zeolite material is colored green. On the blue plane a shadow projection of the sample is visible (black). Scale bar is $200 \mathrm{~nm}$. (a) External surface of Ag/NaY. (b) Crosssection showing silver particles both inside and outside the zeolite.

TEM reconstruction agrees with the size of these particles measured with 2D-TEM.

Our second application of 3D-TEM concerns the imaging of mesopores in a zeolite crystal. The micropores of zeolites are often advantageous to induce shape selectivity, but enhanced accessibility is frequently desirable to restrict mass transfer effects and/or allow catalytic conversion of larger molecules. Several approaches have been followed to enhance accessibility; i.e., the development of zeolites with intrinsically larger pores, ${ }^{14}$ delaminated zeolite precursors, ${ }^{15}$ and zeolite nanocrystals. ${ }^{16}$ It has been established that microporous zeolite crystals can be treated (leaching and/or steaming) to generate mesopores that greatly enhance accessibility and thereby catalytic activity and stability. ${ }^{17-21}$ A case in point are zeolite Y (FAU) and mordenite (MOR). For FAU detailed studies both on the channel ${ }^{17}$ and surface structure ${ }^{18}$ and on the mesoporosity ${ }^{19,20}$ have been carried out using high-resolution TEM. Recently, evidence has been put forward that creation of mesopores in MOR leads to catalysts uniquely suitable for aromatics alkylation chemistry. ${ }^{21}$ Here we show 3D-TEM results on the occurrence of mesopores in acidleached mordenite.

$\mathrm{H}$-mordenite, obtained by calcination of an ammonium exchanged Na-mordenite (LZM-5, Amoco, 5.7 wt \% Al), was acid-leached with $1 \mathrm{M} \mathrm{HCl}$ for $1 \mathrm{~h}$ to give an $\mathrm{H}$-mordenite with 2.6 wt \% Al. For electron microscopy a droplet of a colloidal gold suspension (Sigma, $5 \mathrm{~nm}$ gold) was dried on a carboncoated copper grid, thus providing markers for the alignment of the data set. Next, a droplet of a suspension of the leached mordenite in ethanol was dried on this grid. From a representa- 

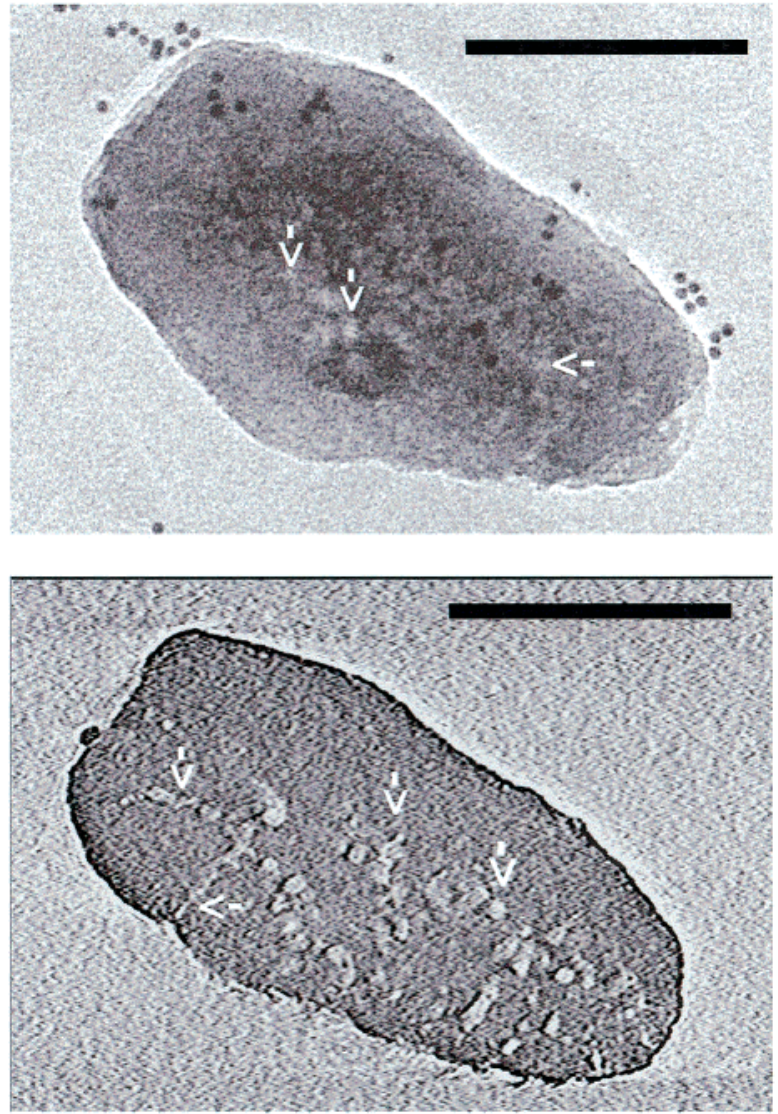

Figure 3. Mesopores in an intact acid-leached H-mordenite crystal. Scale bar is $100 \mathrm{~nm}$. (a) Conventional TEM image indicating the mesopores in the crystallite (white spots, arrows) and several gold beads (black dots, $5 \mathrm{~nm}$ in diameter) on the grid for the alignment. (b) Digital slice $(0.6 \mathrm{~nm}$ thick) through the 3D reconstruction of the crystallite showing the mesopores inside the crystallite (arrows).

tive crystal a tilt series of 141 images was taken from $+70^{\circ}$ to $-70^{\circ}$ with $1^{\circ}$ intervals at a magnification of $20.0 \mathrm{k}$ (pixel size $0.625 \mathrm{~nm}$ ). A representative image of the series is shown in Figure 3a. After alignment, using the gold beads on the grid, a 3 D reconstruction of $270 \times 200 \times 170 \mathrm{~nm}$ with a resolution of $3.3 \mathrm{~nm}$ was calculated. In Figure $3 \mathrm{~b}$ a slice through the reconstruction is shown that reveals the mesopores inside the crystallite with great clarity. In this slice the mesopore structures (3-20 $\mathrm{nm}$ in diameter) are clearly visible although hardly distinguishable in a conventional TEM image (Figure 3a). This is because the surrounding material superimposes on the mesopore in the conventional image and obscures valuable information. Because the 3D-TEM data are obtained from an intact crystal, all visible pores are due to the acid-leaching procedure. With ultramicrotomy the crystals show very often fractures due to the cutting, ${ }^{20}$ which makes the interpretation of the TEM images more difficult.
From the presented results we conclude that 3D-TEM is a unique tool with very high potential for the characterization of solid materials. This is illustrated by the visualization of 3D information with nanometer scale resolution on the interior structure of intact crystals of complex materials.

Acknowledgment. Supported by NWO under Grant 98037. The research of A. J. Koster has been made possible by a fellowship of the Royal Netherlands Academy of Arts and Sciences (KNAW). We thank D. C. Koningsberger, M. Tromp, and J. A. van Bokhoven for the mordenite sample, J. de Graaf for the $\mathrm{Ag} / \mathrm{NaY}$ sample, T. P. van der Krift and W. A. M. van Maurik for assistance with the electron microscopy and digital image handling, and D. J. Lensveld and K. Kadler for revision of the manuscript.

Supporting Information Available: Moving images of the aligned raw electron microscope data, the reconstruction as a stack of slices (as in Figure 1) and the volume rendering of both the entire crystallite and a cut through the reconstruction (as in Figure 2a,b). This material is available free of charge via the Internet at http://pubs.acs.org.

\section{References and Notes}

(1) de Jong, K. P. Cattech 1998, 2, 87-95.

(2) Wijnhoven, J. E. G. J.; Vos, W. L. Science 1998, 281, 802-804.

(3) Dai, H.; Hafner, J. H.; Rinzler, A. G.; Colbert, D. T.; Smalley, R. E. Nature 1996, 384, 147-150.

(4) Koster, A. J.; Agard, D. A. J. Struct. Biol. 1997, 120, 207-209.

(5) Walz, J.; et al. Mol. Cell 1997, 1, 59-65. $81-85$.

6) Baumeister, W.; Grimm, R.; Walz, J. Trends Cell Biol. 1999, 9 ,

(7) Nitsch, M.; et al. Nat. Struct. Biol. 1998, 5, 855-857.

(8) Ladinsky, M. S.; Mastronarde, D. N.; McIntosh, J. R.; Howell, K. E.; Staehelin, L. A. J. Cell Biol. 1999, 144, 1135-1149. 1992.

(9) Electron Tomography; Frank, J., Ed.; Plenum Press: New York,

(10) Koster, A. J.; et al. J. Struct. Biol. 1997, 120, 276-308.

(11) Creyghton, E. J.; Elings, J. A.; Downing, R. S.; Sheldon, R. A.; van Bekkum, H. Microporous Mater. 1996, 5, 299-307.

(12) Maschmeyer, T.; Rey, F.; Sankar, G.; Thomas, J. M. Nature 1995, $378,159-162$.

(13) Bovin, J.-O. Ultramicroscopy 1996, 62, 277-281.

(14) Freyhardt, C. C.; Tsapatsis, M.; Lobo, R. F.; Balkus, K. J., Jr.; Davis, M. E. Nature 1996, 295, 295-298.

(15) Corma, A.; Fornes, V.; Pergher, S. B.; Maesen, Th. L. M.; Buglass, J. G. Nature 1998, 396, 353-356.

(16) Mintova, S.; Olson, N. H.; Valtchev, V.; Bein, T. Science 1999, 283, 958-960.

(17) Sasaki, Y.; Suzuki, T.; Ikuhara, Y. J. Am. Ceram. Soc. 1995, 78, 1411-1413

(18) Alfredsson, V.; Ohsuna, T.; Terasaki, O.; Bovin, J.-O. Angew. Chem., Int. Ed. Engl. 1993, 32, 1210-1213.

(19) Sasaki, Y.; Suszuki, T.; Takamura, Y.; Saji, A.; Saka, H. J. Catal. 1998, 178, 94-100.

(20) Choi-Feng, C.; Hall, J. B.; Huggins, B. J.; Beyerlein, R. A. J. Catal. 1993, 140, 395-405.

(21) Meima, G. R. Cattech 1998, 2, 5-12. 